\title{
Expression of carbonic anhydrase IX (CAIX) in malignant mesothelioma. An immunohistochemical and immunocytochemical study
}

\author{
L. CAPKOVA ${ }^{1, *}$, L. KOUBKOVA ${ }^{2}$, R. KODET ${ }^{1}$ \\ 1Department of Pathology and Molecular Medicine, University Hospital Motol and 2nd Faculty of Medicine, Charles University, V Uvalu \\ 84, 15006 Prague 5, Czech Republic; ${ }^{2}$ Department of Pneumology, University Hospital Motol, V Uvalu 84, 15006 Prague 5, Czech Republic
}

${ }^{*}$ Correspondence: linda.capkova@lfmotol.cuni.cz

Received February 5, 2013 / Accepted May 2, 2013

\begin{abstract}
Malignant mesothelioma is an aggressive tumor with a poor prognosis. Carbonic anhydrase IX (CAIX) is a membranously located metalloenzyme involved in $\mathrm{pH}$ homeostasis with influence on regulation of cell proliferation, oncogenesis and tumor progression. Much attention has been paid recently to carboanhydrases and their inhibitors as they offer an opportunity for both developing novel anticancer drugs, as well as diagnostic and prognostic tools.

This study was designed to assess the expression of CAIX in malignant pleural and peritoneal mesotheliomas, their benign counterparts, and in pleural effusions from patients with malignant mesothelioma, metastatic carcinoma or a benign disease. Tissue blocks from 51 malignant mesotheliomas of pleura (47 cases; 41 epithelioid, 2 biphasic, 4 sarcomatoid) and peritoneum (4 cases; all epithelioid), 14 cases with normal or reactive pleural tissue, and 19 cell blocks were analyzed. CAIX expression was determined using immunohistochemistry and its membranous immunoreactivity was semiquantitatively evaluated. Specimens were divided into five subgroups according to the staining pattern and intensity.

Overall, 92.2\% (47/51) of mesotheliomas expressed CAIX. All epithelioid mesotheliomas showed CAIX positivity, which was predominantly strong and diffuse $(73.3 \%, 33 / 45)$. Sarcomatoid mesotheliomas and sarcomatoid areas in biphasic mesotheliomas were negative. A strong diffuse staining was observed in all cases of normal mesothelia. In pleural effusions, CAIX expression was observed in malignant cells as well as in benign mesothelial cells.

In conclusion, CAIX is expressed virtually in all mesotheliomas except for sarcomatoid subtype, and in benign mesothelia. There are probably more mechanisms of CAIX overexpression than hypoxia-induced in malignant mesothelioma, with the influence of other tissue specific transcription or growth factors depending on the type of the cell lineage. CAIX immunoreactivity is not a reliable diagnostic marker for distinguishing malignant cells from benign mesothelia in pleural effusions. Nevertheless, our data support the potential use of therapeutics targeting CAIX in patients with advanced mesothelioma.
\end{abstract}

Key words: carbonic anhydrase IX, CAIX, malignant mesothelioma, pleura, immunohistochemistry, immunocytochemistry

Malignant pleural mesothelioma (MPM) is the most frequent pleural neoplasm [1]. Its incidence is increasing in the majority of countries and is predicted to peak in the next 10 years [2]. MPM is an aggressive tumor with a poor prognosis. Mortality rate reaches practically 100\% with

Abbreviations: CAIX, carbonic anhydrase IX; MPM, malignant pleural mesothelioma; HIF- $1 \alpha$, hypoxia-inducible factor $1 \alpha$; CA, carbonic anhydrase; HRE, hypoxia response element; VHL, von Hippel-Lindau; CRCC, clear cell renal cell carcinoma; RT-PCR, reverse transcription polymerase chain reaction; ELISA, enzyme-linked immunosorbent assay a median overall survival without treatment of approximately 6 months [3]. The majority of patients are candidates for chemotherapy, either as a single modality treatment or in a combination with other approaches, while in only a small proportion of patients diagnosed with MPM radical surgery with extrapleural pneumonectomy is feasible [4]. Because nearly all MPM patients progress during or after first-line treatment, second-line therapies are being increasingly used. However, there are no standardized therapeutic approaches for second-line chemotherapy and the role and type of these procedures still remain to be established [5]. This 
area poses an ideal field for testing new chemotherapeutic agents, including antiangiogenic drugs, small molecules or monoclonal antibodies that target different pathways at the molecular level [6].

Hypoxia has been recognized as an important factor associated with the growth of a wide range of solid tumors, including malignant mesothelioma [7]. At the molecular level, hypoxia regulates various cellular processes, inclusive cell proliferation, apoptosis, angiogenesis, immunosurveillance, glycolysis, $\mathrm{pH}$ control, adhesion and migration, as well as tumor cell immortalization, invasion and metastasis [8]. There are over 50 genes upregulated under hypoxic conditions via hypoxia-inducible factor $1 \alpha$ (HIF-1a), including the family of carbonic anhydrases (CA) [9]. This group of enzymes comprises 15 zinc metalloenzymes known to date, which are involved in the $\mathrm{pH}$ regulation through the catalysis of conversion of carbon dioxide to bicarbonate and proton. They differ mainly in their catalytic activity, tissue distribution, and subcellular localization [10]. One of the isoforms, the transmembrane carboanhydrase IX (CAIX) isoenzyme, was first described in the HeLa cancer cell line and initially indifferently named the Membrane antigen MN [11]. It is functionally implicated in the tumor progression as a pro-survival factor protecting tumor cells from hypoxia and acidosis via its capability to regulate $\mathrm{pH}$. The association with hypoxia has been linked to the fact that CAIX promoter contains a hypoxia response element (HRE) that is targeted by the transcription factor HIF-1 $\alpha$ [12]. In most instances, the CAIX expression pattern correlates with hypoxia as measured by HIF-1 $\alpha$ protein expression or with oxygen electrode, and therefore, CAIX has been proposed to serve as a surrogate marker of hypoxia [13].

The physiological expression of CAIX is confined to only a few non-neoplastic adult human tissues with distribution restricted to highly specialized cells that are not always hypoxic or in an acidic environment. The only tissues persistently

Table 1. An overview of types and numbers of specimens included in the study

\begin{tabular}{|c|c|}
\hline Specimen type - bioptic material & $\mathrm{N}(\%)$ \\
\hline epithelioid & $41(63.1)$ \\
\hline sarcomatoid & $4(6.2)$ \\
\hline biphasic & $2(3.1)$ \\
\hline Peritoneal malignant mesothelioma (epithelioid) & $4(6.2)$ \\
\hline Normal mesothelium & $7(10.7)$ \\
\hline Reactive mesothelium/pleuritis & $7(10.7)$ \\
\hline Total & $65(100.0)$ \\
\hline Specimen type - cell blocks & $\mathrm{N}(\%)$ \\
\hline Malignant effusion in patients with MPM & $6(31.6)$ \\
\hline Malignant effusion in patients with carcinoma & $6(31.6)$ \\
\hline Reactive benign pleural effusion & $7(36.8)$ \\
\hline Total & $19(100.0)$ \\
\hline
\end{tabular}

Abbreviations: $\mathrm{MPM}=$ malignant pleural mesothelioma expressing CAIX protein during human development are the coelomic epithelium (mesothelium) and its remnants, some epithelia in the gastrointestinal system, and the cells located at the sites previously identified as harboring stem cells, e. g. in the skin and large intestine [14]. An ectopic CAIX expression was described in a wide variety of malignant cell lines and tumor types, especially hypoxic solid tumors derived from uterine cervix, ovary, kidney, lung, oesophagus, breast, colon and others, where it has been used as an endogenous marker of hypoxia [15] or an indicator of poor prognosis [10]. With a few exceptions, CAIX shows focal perinecrotic expression in hypoxic regions in a variety of non-von Hippel-Lindau (VHL)-associated tumors $[12,16]$.

Only little is known about the role of CAIX in the development of malignant mesothelioma and its immunohistochemical staining pattern. In order to gain more insight into the potential role of CAIX in pleural and also peritoneal cavity, immunohistochemical and immunocytochemical expression of this marker was investigated in various histological subtypes of malignant pleural and peritoneal mesothelioma, non-neoplastic pleural samples, and in cytological samples of malignant and benign pleural effusions. Better understanding of the CAIX expression profile in these tissues may potentially lead to the use of innovative treatment approaches in patients with this malignancy.

\section{Patients and methods}

Patients and controls. 51 cases of pleural and peritoneal mesotheliomas and 14 non-neoplastic lesions (specified in Table 1) were retrieved from the surgical pathology files of the Department of Pathology and Molecular Medicine, University Hospital Motol, Prague, Czech Republic, from the years 1999 to 2012. Written informed consent was acquired from all participants before they were included in the study. The diagnoses were confirmed previously in another study (data not published) using a panel of immunohistochemical stains that included 5 positive (D2-40, CK5/6, calretinin, WT1 and HBME-1) and 4 negative (CEA, MOC-31, Ber-EP4 and B72.3) mesothelial markers. There were 47 patients with MPM (25 male, 22 female) with the mean age of 61.3 years (age range 41 to 87 years). In addition, 4 patients with malignant peritoneal mesotheliomas ( 1 male, 3 female, mean age 53.3 years, age range 30 to 73 years), 14 biopsies either with normal (patients with spontaneous pneumothorax) or reactive hyperplastic mesothelium (samples from patients with organizing fibrinous and chronic fibroproductive pleuritis), and cell blocks of pleural effusion samples from 19 patients with histologically confirmed diagnosis of MPM, metastatic carcinoma (3 lung, 2 ovarian and 1 colorectal adenocarcinoma) or reactive pleuritis were included (Table 1). All the biopsies and cytological specimens enrolled into this study were taken as diagnostic samples prior to any chemo- or radiation therapy.

Immunohisto- and immunocytochemisty. Formalinfixed, paraffin-embedded, $4-\mu \mathrm{m}$ thick tissue sections were 
deparaffinized in xylene, rehydrated in a descending series of ethanol, and stained with the non-commercial mouse monoclonal anti-CAIX antibody raised to the external Nterminal domain of CAIX protein using described previously methods (clone M75, kindly provided by Jan Zavada, Institute of Molecular Genetics, Academy of Sciences, Czech Republic) $[11,17]$. Standard avidin-biotin immunoperoxidase technique with no epitope retrieval at an antibody dilution of 1:100 was used. The antigen-antibody complexes were visualized using Histofine Kit (Nichirei Bioscience Inc., Japan) with diaminobenzidine (DAKO) as the chromogen. The tissue sections were counterstained with Harris' hematoxylin, dehydrated, cleared in xylene and mounted. Clear cell renal cell carcinoma cases (CRCC) served as positive controls, and negative control experiments were performed appropriately.

Evaluation of CAIX staining. Immunostaining results were evaluated independently by two pathologists (LC, RK). Only membranous staining was considered positive for the evaluation of CAIX expression. The proportion and distribution pattern (absent, $0 \%$; focal/patchy, less than $50 \%$; or diffuse, $\geq 50 \%$ ) of CAIX-positive cells was estimated and the intensity of staining was scaled from absent to weak (weaker than positive CRCC control) and strong (of the same intensity as positive CRCC control). Thus, 5 categories of CAIX expression were set: negative, weak focal, weak diffuse, strong focal and strong diffuse staining. All categories with CAIX immunostaining, whether strong or weak and focal or diffuse, were considered positive, and therefore grouped collectively.

Statistical analysis. All statistical test were performed with the statistical program StatView (Abacus Concept, Berkeley,
California). Comparison between the morphological findings and CAIX expression was performed by using the KruskalWallis H-test. The level of statistical significance was set at $\mathrm{p}<0.05$.

\section{Results}

All 51 tumor and 14 non-neoplastic bioptic samples were informative for the determination of CAIX expression. Overall, 92.2\% (47/51) of mesothelioma cases expressed CAIX. All epithelioid mesotheliomas (pleural and peritoneal) showed at least weak focal $(8.9 \%, 4 / 45)$, but predominantly strong diffuse (73.3\%, 33/45) staining with CAIX antibody (Fig. 1A and 1B). Biphasic mesothelioma showed positivity in its epithelioid regions (Fig. 1D). Only 4 cases without any CAIX expression $(7.8 \%, 4 / 51)$ were those of sarcomatoid histotype. Table 2 shows the immunohistochemical results of CAIX expression in mesothelial lesions in summary.

The positivity of CAIX in MPM and peritoneal mesothelioma was diffuse or disperse, nevertheless without any association either with necrosis (Fig. 1C, asterisk) or dependance on the distance from the vessels (Fig. 2A, arrow). In sarcomatoid areas of biphasic mesothelioma and in tumors with dedifferentiated areas such as giant bizzare cells or small areas with transition into sarcomatoid pattern insufficient for the diagnosis of biphasic type, the staining typical for the epithelioid areas was not present (Fig. 1D, asterisk). In peritoneal mesotheliomas, CAIX expression was observed rather in papillary and tubular formations than in solid nests. Tumor-associated stromal and inflammatory cells were negative.

Table 2. CAIX grading system and summary of CAIX expression in bioptic samples

\begin{tabular}{lcccccc}
\hline \multirow{2}{*}{ Histological diagnosis } & \multicolumn{5}{c}{ CAIX expression - No. of cases (\%) } \\
\cline { 2 - 6 } & Positive total & Strong diffuse & Strong focal & Weak diffuse & Weak focal & Negative \\
\hline MM total & $47 / 51(92.2)$ & $33(64.7)$ & $7(13.7)$ & $3(5.9)$ & $4(7.8)$ & $4(7.8)$ \\
$\quad$ epithelioid & $45 / 45(100.0)$ & $33(73.3)$ & $5(11.1)$ & $3(6.7)$ & $4(8.9)$ & 0 \\
biphasic & $2 / 2(100.0)$ & 0 & $2(100.0)$ & 0 & 0 & 0 \\
sarcomatoid & $0 / 4(0)$ & 0 & 0 & 0 & $1(14.3)$ & $1(14.3)$ \\
RM/pleuritis & $6 / 7(85.7)$ & $3(42.8)$ & $2(28.6)$ & 0 & 0 \\
NM & $7 / 7(100)$ & $7(100)$ & 0 & 0 & 0 \\
\hline
\end{tabular}

Abbreviations: CAIX = carbonic anhydrase IX; $\mathrm{MM}=$ malignant mesothelioma; $\mathrm{RM}=$ reactive mesothelia; $\mathrm{NM}=$ normal mesothelia

Table 3. CAIX grading system and summary of CAIX expression in pleural effusions

\begin{tabular}{lcccccc}
\hline \multirow{2}{*}{ Cytological diagnosis } & \multicolumn{5}{c}{ CAIX expression - No. of cases (\%) } \\
\cline { 2 - 6 } & Positive total & Strong diffuse & Strong focal & Weak diffuse & Weak focal & Negative \\
\hline Malignant effusion in patients with MM & $6 / 6(100.0)$ & $5(83.3)$ & 0 & 0 & $1(16.7)$ & 0 \\
Malignant effusion in patients with carcinoma & $4 / 6(66.7)$ & 0 & $2(33.3)$ & 0 & $2(33.3)$ & $2(33.3)$ \\
Benign effusion & $6 / 7(85.6)$ & $3(42.8)$ & $2(28.6)$ & 0 & $1(14.3)$ & $1(14.3)$ \\
\hline
\end{tabular}

Abbreviations: CAIX = carbonic anhydrase IX; $\mathrm{MM}=$ malignant mesothelioma 

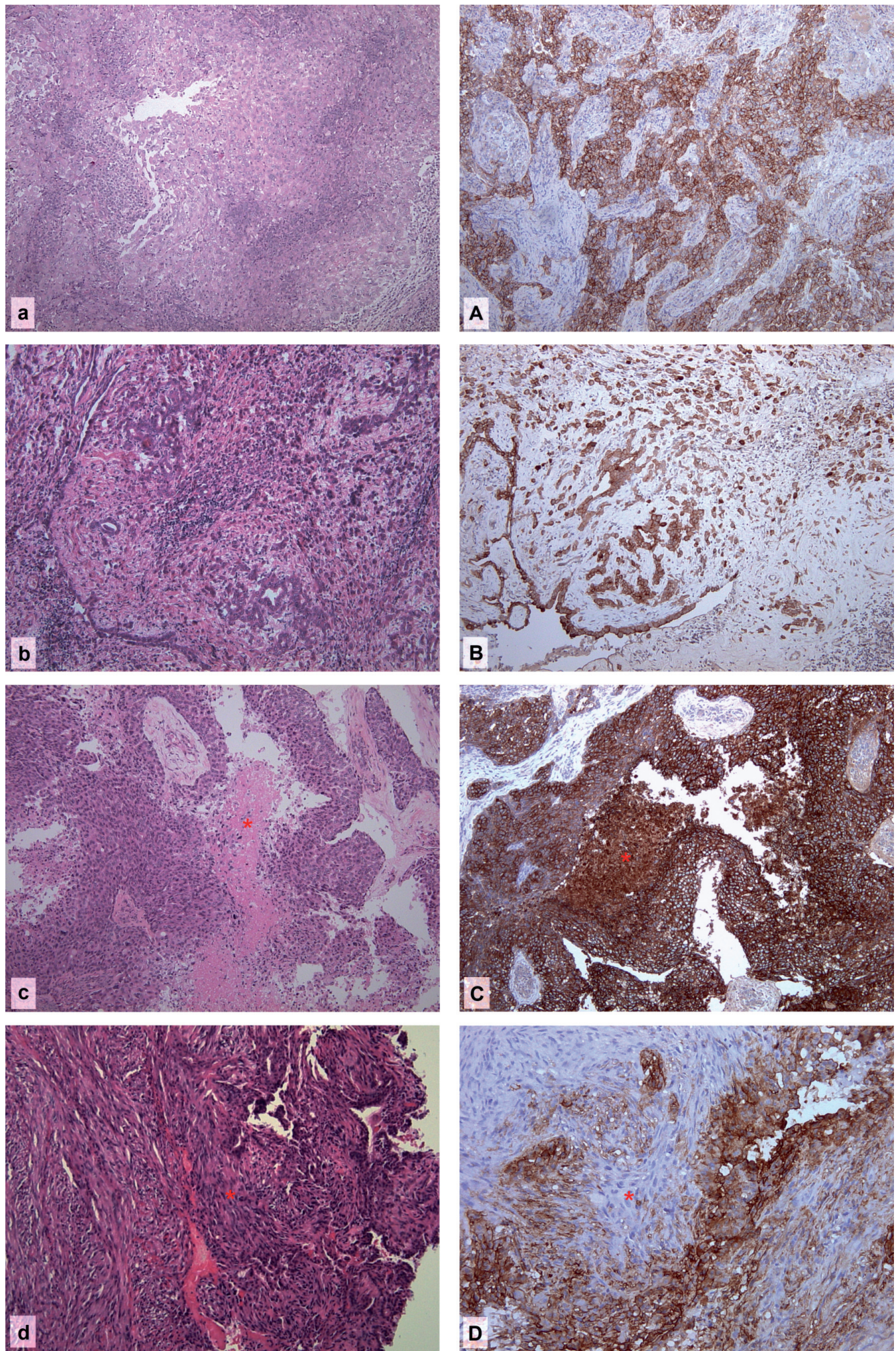

Fig. 1 Haematoxylin and eosin and CAIX staining in malignant mesotheliomas. (a) Epithelioid mesothelioma with fibrovascular cores and an inflammatory infiltrate. (A) Diffuse strong membranous CAIX reactivity. (b) Epithelioid mesothelioma with tumor cells dissociated in the stroma. (B) Diffuse strong membranous CAIX reactivity. (c) Epithelioid mesothelioma with necrosis (asterisk). (C) Diffuse and strong CAIX expression without any association with necrotic areas. (d) Biphasic mesothelioma with sarcomatoid areas (asterisk). (D) No CAIX expression is observed in sarcomatoid areas (asterisk), whereas tumor cells with epithelioid appearance retain CAIX positivity. (Original magnification $\times 100$ ). 

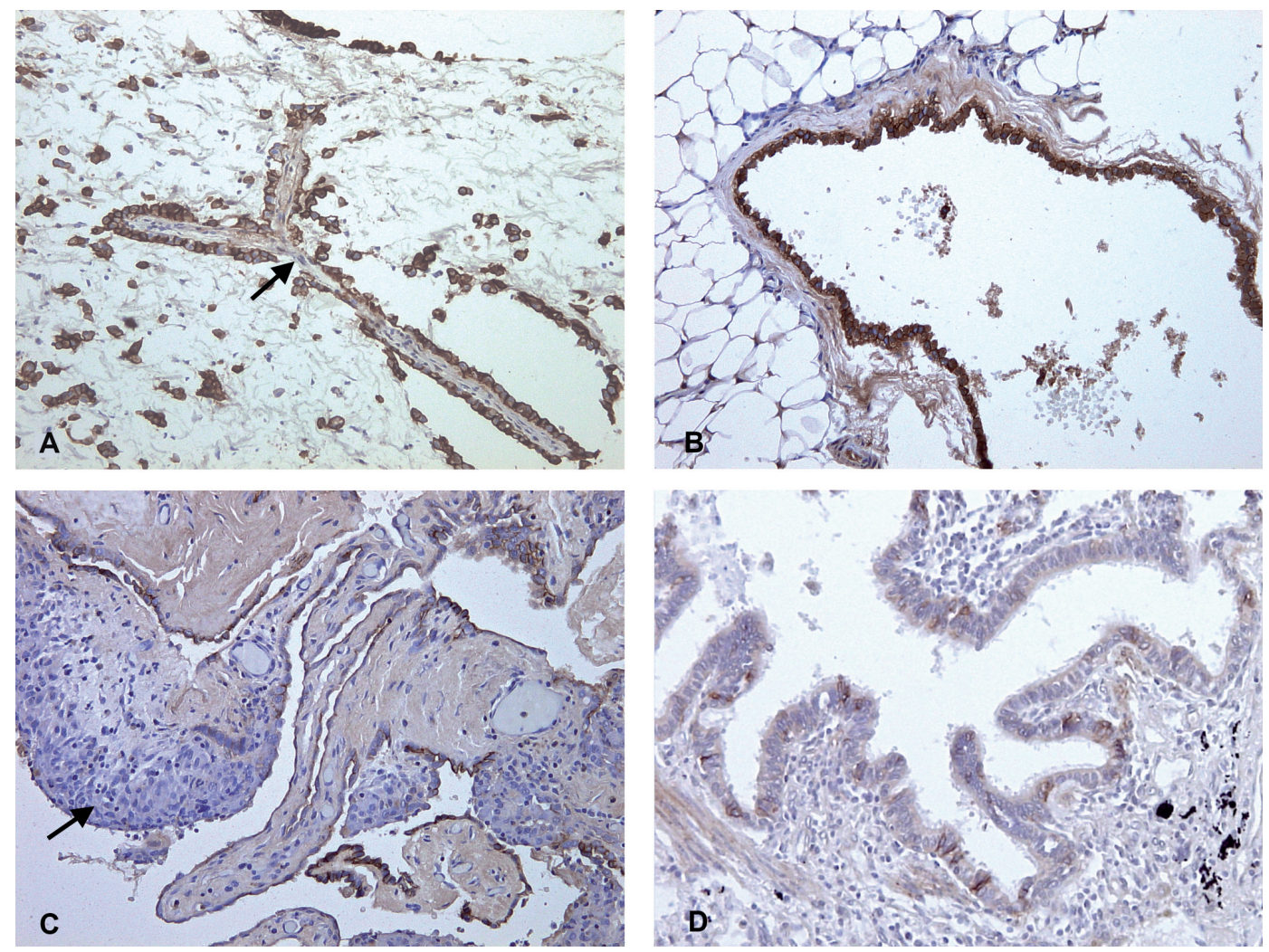

Fig. 2 CAIX immunohistochemistry in the lung and pleural tissues. (A) Membranous positivity in malignant pleural mesothelioma in the direct proximity of a vessel (arrow). (B) Non-neoplastic mesothelial cells showing strong diffuse membranous positivity. (C) Reactively proliferating hyperplastic mesothelial cells with strong and diffuse staining and mesothelial cells entrapped in granulation tissue with no CAIX expression (arrow). (D) Membranous staining seen in some of the bronchial columnar epithelial cells. (Original magnification $\times 200$ ).

In samples with adjacent lung tissue present in the specimens, membranous staining was identified in some of bronchial columnar epithelial cells (Fig. 2D), as was already described elsewhere [14]. Pneumocytes, alveolar macrophages, as well as stromal cells were negative.

In all samples with normal mesothelium $(100 \%, 7 / 7)$, the mesothelial cells showed strong diffuse membranous positivity (Fig. 2B). Reactively proliferating mesothelial cells on pleural surface in chronic fibroproductive pleuritis were stained strongly $(71.4 \%, 5 / 7)$ and mostly diffusely as well $(42.8 \%, 3 / 7)$. Only benign reactive hyperplastic mesothelial cells entrapped within granulation tissue of an organizing fibrinous pleuritis showed no or only a weak focal CAIX expression $(28.6 \%$, 2/7) (Fig. 2C, arrow). No statistically significant differences of immunohistochemical CAIX positivity between MPM and non-tumorous mesothelia were observed $(\mathrm{p}=0.8991)$.

CAIX reactivity in pleural effusions is depicted in Table 3. Membranous CAIX positivity mostly of strong diffuse pattern was observed in all $(100 \%, 6 / 6)$ malignant mesotheliomas (Fig. 3D). $66.7 \%$ (4/6) of carcinomas and $85.7 \%$ (6/7) of reactive mesothelial cells stained as well, with strong CAIX reactivity in $33.3 \%(2 / 6)$ of carcinomas (Fig. 3C), and $73.2 \%(5 / 7)$ of benign effusions (Fig. 3A, arrow) respectivelly. There was no statistically significant difference of the CAIX expression between these cytologically distinct groups $(\mathrm{p}=0.6197)$.

\section{Discussion}

The current study aimed to test malignant mesotheliomas and benign mesothelial cells in detail with regard to sparse data on CAIX reactivity in the literature. Until recently, expression of CAIX was briefly noted immunohistochemically in 2 samples of nonneoplastic mesothelial cells and 8 cases of MPM [18], and via reverse transcription polymerase chain reaction (RT-PCR) in pleural effusions from 8 patients with MPM [19]. Lately, Ramsey et al. [20] reported prevailing immunohistochemical CAIX expression in 19 MPMs and 4 cases of non-neoplastic mesothelial cells (one case of mesothelial hyperplasia, 3 samples of normal pleural mesothelium), nevertheless all these reports lack any closer specifications of staining patterns and intensity. In other solid tumors investigated previously, heterogeneous CAIX immunostaining with the highest intensity in areas adjacent to the necrosis was noted $[16,21,18]$. The observations are different in CRCC, in which 

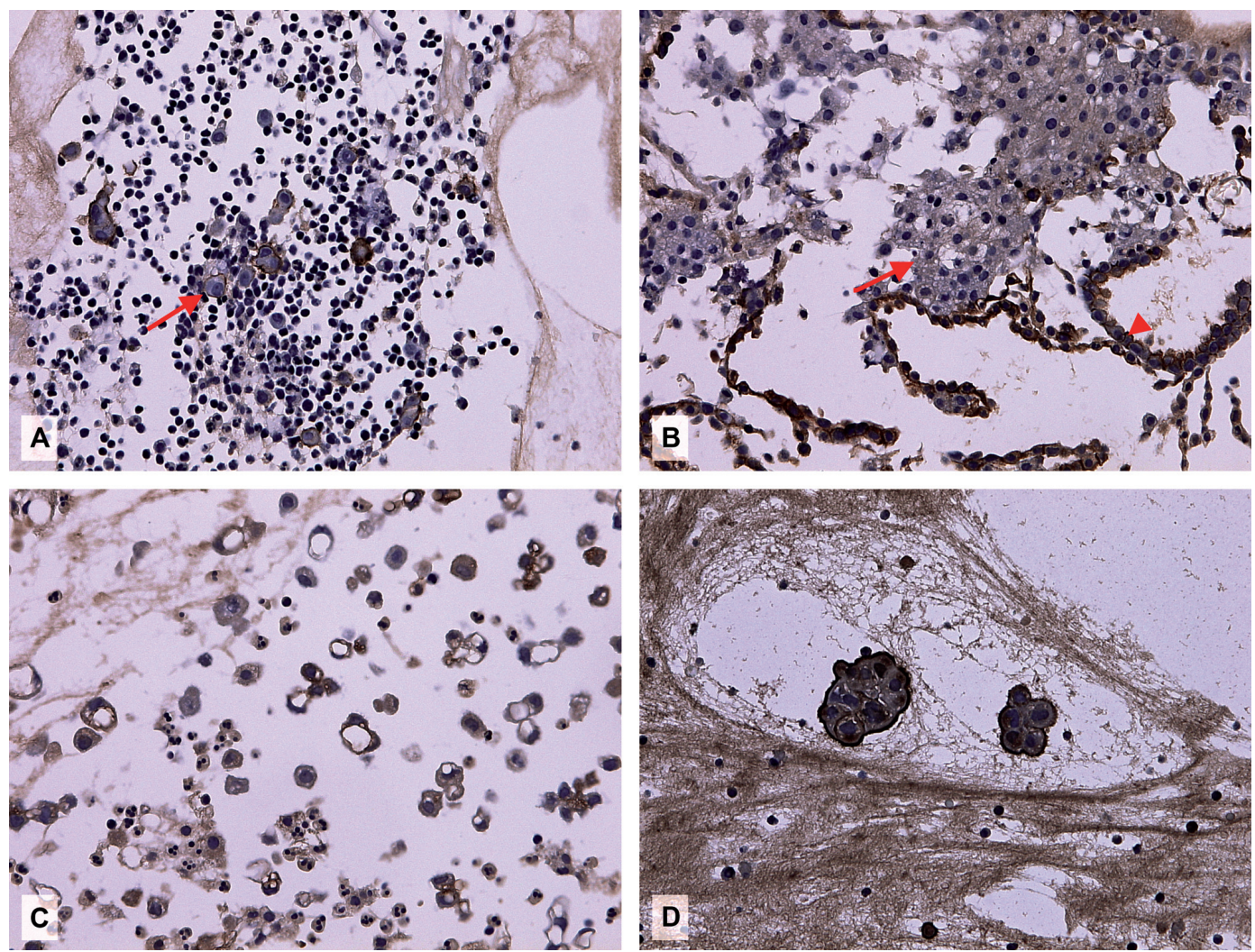

Fig. 3 Immunocytochemical CAIX reactivity in cell blocks from pleural effusions. (A) Membranous positivity in benign reactive mesothelial cells (arrow) from a patient with cardiac failure. (B) CAIX immunostaining of reactive mesothelial cells (arrowhead) and negativity of tumor cells (arrow) in malignant effusion from a patient with metastatic colorectal adenocarcinoma. (C) Positive membranous staining in atypical vacuolized malignant cells in a patient with underlying serous ovarian carcinoma. (D) Membranous expression of strong intensity in malignant mesothelial cells in a patient with malignant mesothelioma. (Original magnification $\times 400)$.

a uniformly diffuse staining pattern is caused by constitutive up-regulation of HIF- $1 \alpha$ and loss of regulation by hypoxia due to the inactivity of VHL gene [9].

In the present study, diffuse staining and strong staining intensity was observed in the majority of mesotheliomas (64.7\%), independently on the presence of necrosis and unrelated to vessel proximity. Our finding is similar to observations made by other authors in CRCC, despite the fact that VHL mutations do not seem to play any significant role in mesothelioma oncogenesis $[22,23]$. Of special concern was the staining pattern in biphasic mesotheliomas and in epithelioid mesotheliomas with dedifferentiated areas (presence of anaplasia with giant bizzare cells or zones of transition into sarcomatoid pattern), where the staining typical for the epithelioid areas was lost. Sarcomatoid mesotheliomas were uniformly negative. A similar phenomenon, where mesothelioma shows a decreased expression of epithelial and mesothelial epitopes in its sarcomatoid component, is apparent in immunostaining pattern using other so called mesothelial markers [24].

Interestingly, the percentage of CAIX-positive mesotheliomas and the intensity of staining in the presented analysis was somewhat lower than described by Ramsey et al., who reported strong and diffuse positivity in all cases of pleural mesothelioma [20]. This could be caused by using different clones of anti-CAIX antibody (M75 vs. TH22 by Leica), by different interpretation of sarcomatoid pattern in biphasic cases in both studies, and by the fact that 4 sarcomatoid mesotheliomas were included in our series. All epithelioid mesotheliomas were positive in the above cited and in our study. However, Ramsey et al. reported no appreciable difference in reactivity between epithelioid and biphasic subtypes of MPM and did not investigate any purely sarcomatoid mesotheliomas. Ramsey et al. also proposed CAIX being a novel mesothelial marker, but regarding its positivity in non-neoplastic mesothelium and in many other malignant tumors affecting lung and pleura $[16,18,21]$, it seems to have no practical value in the light of differential diagnosis.

The CAIX expression pattern in non-neoplastic mesothelia also deserves attention. There is a similarly strong diffuse membranous CAIX positivity in virtually all non-neoplastic mesothelial cells (normal mesothelium and reactive mesothelial hyperplasia), as long as the cells retain epithelioid 
appearance and reactively proliferate on the pleural surface. This is consistent with the previous data published by Ramsey [20]. However, the expression of CAIX clears off under condition of serosal injury when the reactive hyperplastic mesothelial cells get entrapped within fibrin and granulation tissue in the originally submesothelial layer in case of an organizing pleuritis. This could coincide with the ultrastructural observations in healing serosa where the mesothelial cells adapt a different, myofibroblast-like phenotype [25].

It is evident that hypoxic pathway is the main regulator of CAIX expression in most of the solid tumors as shown in numerous previous studies $[9,12,10]$ and therefore, positivity in hypoxic and perinecrotic areas would be expected in malignant mesothelioma. Our findings show mostly strong and diffuse CAIX expression in mesotheliomas without any significant tumor necrosis as well as in benign mesothelial tissue. Additionally, no HIF-1a expression was described in non-neoplastic mesothelia previously [7]. This would suggest the possibility that regulatory mechanisms and transcriptional factors other than hypoxia modulate CAIX expression in mesothelial cells. Liao et al. [14] also showed recently that the expression of CAIX in normal adult tissues might not be induced by hypoxia only, but seems to be related to functional status and to the type of the cell of origin of the relevant tissue.

Recently, two studies evaluated the role of CAIX as a new marker for the detection of malignant cells in pleural effusions. Liao et al. [26] used CAIX rabbit polyclonal antibody (Santa Cruz) for the immunohistochemical detection of CAIX expression in 58 benign and 47 malignant effusions, which represented dissemination of various malignancies in pleural cavity, but none was due to the dissemination of MPM. The CAIX protein was significantly expressed in $63.8 \%$ of malignant effusions and its expression was not observed in non-neoplastic cytologies. The authors also used the category of probable malignant pleural effusions, which were defined as ,effusions occuring in a patient with a known primary malignancy and a negative pleural fluid cytology finding". In this group, they observed CAIX positivity in $15.8 \%$ of cases predominantly in cells resembling reactive cells of benign appearance. The authors interpreted this finding as an attribute of malignancy and concluded that CAIX might be a helpful complementary marker of malignant cells in pleural effusions. Analogous findings and conclusions were made by the same authors in a recent study combining immunohistochemistry and enzyme-linked immunosorbent assay (ELISA) [27]. Li et al. [19], who used RT-PCR for qualitative detection of MN/ CA9 mRNA expression, came with similar results. In their study, the majority (89.8\%) of malignant pleural effusions (including 7 of 8 mesotheliomas), as well as of cytologically negative effusions from cancer patients (72.2\%) was positive, whereas only $8.3 \%$ of effusions from control patients with a benign disease showed positivity. The presented study of CAIX expression in cell blocks, however, leads to a different conclusion. In malignant effusions (MPM and carcinoma)
CAIX was expressed in $83.3 \%(10 / 12)$ and in benign effusions in $85.7 \%(6 / 7)$ of cases $(p=0.6197)$. Based on our results using the M75 antibody for immunohistochemical identification of CAIX, we are convinced that CAIX immunoreactivity in pleural effusions should not be regarded as a marker of malignancy, since we demonstrated its frequent positivity in normal and reactive mesothelial cells in benign pleural effusions as well as in histologic samples to be a common phenomenon rather than a discriminating factor.

During the last decade, it has become evident, that CAs and their inhibitors offer an opportunity for both developing novel drugs [28], as well as diagnostic and prognostic tools [29]. Development of new CAIX-specific antibodies directed against the catalytic domain of CAIX [30] might serve as a model for preclinical studies of immunotherapeutic strategies for the treatment of tumors other than CRCC, which have not been explored so far. However, given the fact that CAIX is expressed in some normal human organs and tissues including mesothelial cells as was shown in this study, the development of isozyme-specific organ-selective inhibitors is necessary to keep the therapeutic toxicity to a minimum. These drugs might be potentially applied directly to pleural cavity with similar risks of pleural damage and consequences for the mesothelial layer as in talcopleurodesis [31].

\section{Conclusions}

The immunohistochemical positivity of CAIX was observed in virtually all malignant mesotheliomas as well as in normal and reactive mesothelial cells examined in this study, with some exceptions described earlier. Our data support the concept of selective CAIX inhibition as a potential therapeutic target for patients with advanced malignant mesothelioma. CAIX expression in various malignant as well as in benign mesothelia does not allow CAIX to serve as a reliable diagnostic marker of malignancy in pleural effusions. Although CAIX is an interesting target for novel approaches in antitumor treatment, its exact role in tumor growth and progression remains to be investigated, particularly in malignant mesothelioma, where its expression seems to be probably more closely related to the cell line of origin than in other malignancies.

Acknowledgements: The study was supported by the project of Ministry of Health, Czech Republic for conceptual development of research organization 00064203 (University Hospital Motol, Prague, Czech Republic) and by the project OPPK CZ.2.16/3.1.00/24022.

The authors thank E. Axmannova and S. Pospisilova for their skillful technical assistance.

\section{References}

[1] TRAVIS WD, BRAMBILLA E, MÜLLER-HERMELINK HK, HARRIS CC, editors. Pathology and genetics of tumors of the lung, pleura, thymus and heart. Lyon: IARC Press, 2004. 
[2] ROBINSON BW, LAKE RA. Advances in malignant mesothelioma. N Engl J Med 2005; 353: 1591-603. http://dx.doi. org/10.1056/NEJMra050152

[3] ISMAIL-KHAN R, ROBINSON LA, WILLIAMS CC, JR., GARRETT CR, BEPLER G, et al. Malignant pleural mesothelioma: a comprehensive review. Cancer Control 2006; 13: 255-63.

[4] CERESOLI GL, GRIDELli C, SANTORO A. Multidisciplinary treatment of malignant pleural mesothelioma. Oncologist 2007; 12: 850-63. http://dx.doi.org/10.1634/ theoncologist.12-7-850

[5] CERESOLI GL, ZUCALI PA, GIANONCELLI L, LORENZI E, SANTORO A. Second-line treatment for malignant pleural mesothelioma. Cancer Treat Rev 2010; 36: 24-32. http:// dx.doi.org/10.1016/j.ctrv.2009.09.003

[6] KELLY RJ, SHARON E, HASSAN R. Chemotherapy and targeted therapies for unresectable malignant mesothelioma. Lung Cancer 2011; 73: 256-63. http://dx.doi.org/10.1016/ j.lungcan.2011.04.014

[7] KLABATSA A, SHEAFF MT, STEELE JP, EVANS MT, RUDD RM, et al. Expression and prognostic significance of hypoxia-inducible factor 1alpha (HIF-1alpha) in malignant pleural mesothelioma (MPM). Lung Cancer 2006; 51: 53-9. http://dx.doi.org/10.1016/j.lungcan.2005.07.010

[8] HARRIS AL. Hypoxia--a key regulatory factor in tumor growth. Nat Rev Cancer 2002; 2: 38-47. http://dx.doi. org/10.1038/nrc704

[9] POTTER C, HARRIS AL. Hypoxia inducible carbonic anhydrase IX, marker of tumor hypoxia, survival pathway and therapy target. Cell Cycle 2004; 3: 164-7. http://dx.doi. org/10.4161/cc.3.2.618

[10] PASTOREKOVA S, PARKKILA S, PASTOREK J, SUPURAN CT. Carbonic anhydrases: current state of the art, therapeutic applications and future prospects. J Enzyme Inhib Med Chem 2004; 19: 199-229. http://dx.doi.org/10.1080/ 14756360410001689540

[11] PASTOREKOVA S, ZAVADOVA Z, KOSTAL M, BABUSIKOVA O, ZAVADA J. A novel quasi-viral agent, MaTu, is a two-component system. Virology 1992; 187: 620-6. http:// dx.doi.org/10.1016/0042-6822(92)90464-Z

[12] WYKOFF CC, BEASLEY NJ, WATSON PH, TURNER KJ, PASTOREK J, et al. Hypoxia-inducible expression of tumor-associated carbonic anhydrases. Cancer Res 2000; 60: 7075-83.

[13] PASTOREKOVA S, PARKKILA S, ZAVADA J. Tumor-associated carbonic anhydrases and their clinical significance. Adv Clin Chem 2006; 42: 167-216. http://dx.doi.org/10.1016/ S0065-2423(06)42005-9

[14] LIAO SY, LERMAN MI, STANBRIDGE EJ. Expression of transmembrane carbonic anhydrases, CAIX and CAXII, in human development. BMC Dev Biol 2009; 9: 22. http://dx.doi. org/10.1186/1471-213X-9-22

[15] ROBERTSON N, POTTER C, HARRIS AL. Role of carbonic anhydrase IX in human tumor cell growth, survival, and invasion. Cancer Res 2004; 64: 6160-5. http://dx.doi. org/10.1158/0008-5472.CAN-03-2224

[16] KIM SJ, RABBANI ZN, VOLLMER RT, SCHREIBER EG, OOSTERWIJK E, et al. Carbonic anhydrase IX in early-stage non-small cell lung cancer. Clin Cancer Res 2004; 10: 7925-33. http://dx.doi.org/10.1158/1078-0432.CCR-04-0636

[17] ZAVADA J, ZAVADOVA Z, PASTOREKOVA S, CIAMPOR F, PASTOREK J, et al. Expression of MaTu-MN protein in human tumor cultures and in clinical specimens. Int J Cancer 1993; 54: 268-74. http://dx.doi.org/10.1002/ ijc. 2910540218

[18] IVANOV S, LIAO SY, IVANOVA A, DANILKOVITCHMIAGKOVA A, TARASOVA N, et al. Expression of hypoxia-inducible cell-surface transmembrane carbonic anhydrases in human cancer. Am J Pathol 2001; 158: 905-19. http://dx.doi.org/10.1016/S0002-9440(10)64038-2

[19] LI G, PASSEBOSC-FAURE K, FENG G, LAMBERT C, COTTIER M, et al. MN/CA9: a potential gene marker for detection of malignant cells in effusions. Biomarkers 2007; 12: 214-20. http://dx.doi.org/10.1080/13547500601068192

[20] RAMSEY ML, YUH BJ, JOHNSON MT, YELDANDI AV, ZYNGER DL. Carbonic anhydrase IX is expressed in mesothelioma and metastatic clear cell renal cell carcinoma of the lung. Virchows Arch 2011; 460: 89-93. http://dx.doi. org/10.1007/s00428-011-1178-7

[21] LONCASTER JA, HARRIS AL, DAVIDSON SE, LOGUE JP, HUNTER RD, et al. Carbonic anhydrase (CA IX) expression, a potential new intrinsic marker of hypoxia: correlations with tumor oxygen measurements and prognosis in locally advanced carcinoma of the cervix. Cancer Res 2001; 61: 6394-9.

[22] SEKIDO Y, BADER S, LATIF F, GNARRA JR, GAZDAR AF, et al. Molecular analysis of the von Hippel-Lindau disease tumor suppressor gene in human lung cancer cell lines. Oncogene 1994; 9: 1599-604.

[23] JEAN D, DAUBRIAC J, LE PIMPEC-BARTHES F, GALATEAU-SALLE F, JAURAND MC. Molecular changes in mesothelioma with an impact on prognosis and treatment. Arch Pathol Lab Med 2012; 136: 277-93. http://dx.doi. org/10.5858/arpa.2011-0215-RA

[24] LUCAS DR, PASS HI, MADAN SK, ADSAY NV, WALI A, et al. Sarcomatoid mesothelioma and its histological mimics: a comparative immunohistochemical study. Histopathology 2003; 42: 270-9. http://dx.doi.org/10.1046/j.1365-2559.2003.01583. $\underline{\mathrm{x}}$

[25] MUTSAERS SE. The mesothelial cell. Int J Biochem Cell Biol 2004; 36: 9-16. http://dx.doi.org/10.1016/S13572725(03)00242-5

[26] LIAO ND, SHIEH JM, LEE WY. Diagnostic value of metabolic phenotypes in malignant pleural effusions: expression of GLUT1 and CAIX by immunocytochemistry. Cancer Cytopathol 2011; 119: 346-53. http://dx.doi.org/10.1002/ cncy. 20153

[27] LIAO ND, LEE WY. Detection of carbonic anhydrase IX protein in the diagnosis of malignant pleural effusion by enzyme-linked immunosorbent assay and immunocytochemistry. Cancer Cytopathol 2012; 120: 269-75. http://dx.doi. org/10.1002/cncy. 21191

[28] SUPURAN CT. Carbonic anhydrases as drug targets--an overview. Curr Top Med Chem 2007; 7: 825-33. http://dx.doi. org/10.2174/156802607780636690 
[29] Parkkila S. Significance of $\mathrm{pH}$ regulation and carbonic anhydrases in tumor progression and implications for diagnostic and therapeutic approaches. BJU Int 2008; 101 Suppl 4: 16-21. http://dx.doi.org/10.1111/j.1464410X.2008.07643.x

[30] ZATOVICOVA M, JELENSKA L, HULIKOVA A, CSADEROVA L, DITTE Z, et al. Carbonic anhydrase IX as an anticancer therapy target: preclinical evaluation of internalizing monoclonal antibody directed to catalytic domain. Curr Pharm Des 2010; 16: 3255-63. http://dx.doi.org/10.2174/ 138161210793429832

[31] RODRIGUEZ-PANADERO F, MONTES-WORBOYS A. Mechanisms of pleurodesis. Respiration 2012; 83: 91-8. http://dx.doi.org/10.1159/000335419 